\title{
ESSAI DE RÉVISION \\ DES PHYSALOPTÈRES PARASITES DE REPTILES
}

\author{
Par Alain G. CHABAUD
}

\section{Introduction}

Plusieurs importantes révisions ont été publiées sur les Physaloptères : Seurat (1917), Travassos (1920), Irwin-Smith (1921, 1922), Ortlepp (1922, 1937), Schulz (1927), Hill (1940) et Morgan (1942, 1943,1945 et 1947). Ces monographies ont été faites avec le plus grand soin et sont extrêmement précieuses, mais le groupe est tellement riche en espèces, et les espèces sont tellement proches morphologiquement les unes des autres, que les diagnoses restent encore souvent très difficiles.

La plupart des révisions (et surtout la plus récente : celle de Morgan) sont de tendance uniciste et mettent un grand nombre d'espèces en synonymie. La notion habituellement admise est que beaucoup d'espèces ont une morphologie variable, une spécificité parasitaire peu marquée et une aire de distribution géographique extrêmement large. Il est possible que ces notions soient parfaitement valables en ce qui concerne les Physaloptères parasites d'Oiseaux migrateurs ou de Mammifères domestiques, mais, pour les espèces de Reptiles, nous avons été amené peu à peu à une vue un peu différente. La spécificité parasitaire semble large; des auteurs très avertis, tels que Seurat et Ortlepp, qui ont eu un matériel abondant à étudier, n'ont pas hésité à identifier à une même espèce des parasites de Varans, de Caméléons et de Serpents (ex. : Abbreviata paradoxa, sensu Seurat 1924). Au contraire, la notion d'espèces à répartition géographique extrêmement étendue ne semble pas devoir être admise aussi facilement.

Tout d'abord, il est remarquable de constater qu'Ortlepp, ayant étudié un matériel très abondant et varié, provenant du monde entier, n'a jamais identifié personnellement une même espèce de Physaloptère de Reptiles dans des régions géographiques très éloignées. Cette notion d'aire géographique très large pour une même espèce parasite de Reptiles n'est jamais due à un auteur ayant

Ann, de Parasitologie, T. XXXI, N ${ }^{\circ} 1-2-1956$. 
comparé des spécimens géographiquement très différents ; elle est obtenue seulement en compilant les différentes identifications faites par des auteurs variés sur des récoltes particulières. Dans ce cas, l'analyse critique de la bibliographie montre tantôt des erreurs de détermination manifestes, tantôt des identifications sans commentaires, ni descriptions, qui peuvent également recéler des erreurs. Nous verrons par exemple que, pour une espèce telle qu'A. abbreviata, citée de pays très variés, les références concernant l'Amérique du Sud et l'Afrique Orientale sont sùrement fausses, celles du Turkestan douteuses, et que les seules références valables semblent actuellement limitées à la région méditerranéenne occidentale.

Enfin, les difficultés que nous avons rencontrées récemment pour déterminer certains spécimens nous incitent également à une conception pluraliste de la systématique des Physaloptères de Reptiles. En voici deux exemples :

a) Ortlepp, ayant déterminé comme $P$. paradoxa un parasite de Psammophis sibilans (L.) du Soudan, se crut autorisé à mettre en synonymie le $P$. affinis de Gedoelst, décrit précisément chez le même hôte au Congo belge. Baylis, ayant eu l'occasion ultérieurement d'examiner le matériel de Gedoelst, a constaté qu'il ne répondait pas à la description du paradoxa d'Ortlepp. Or, l'examen d'un Physaloptère parasite d'un autre Serpent du Moyen-Congo nous a montré que les parasites avaient bien tous les caractères décrits par Gedoelst et non ceux de paradoxa. Il devient donc évident que, suivant les régions, le même hôte $P$. sibillans peut avoir deux parasites différents et l'espèce de Gedoelst doit être rétablie.

b) Des Physaloptères ont été récoltés à différentes reprises et par différents auteurs chez Varanus exanthematicus (Bosc.). Baylis et Daubney (1923) hésitent entre varani et quadrovaria et concluent à quadrovaria. Kung (1948), au contraire, le détermine comme paradoxa, en constatant pourtant que la structure génitale ne correspond pas à celle qui est décrite pour l'espèce. Ayant un nouveau matériel du même hôte, nous constatons pour la troisième fois les mêmes particularités ; nous croyons donc qu'il devient nécessaire d'individualiser spécifiquement ce parasite. Les Varans africains paraissent avoir ainsi au moins quatre Physaloptères différents : l'un en Afrique du Sud, l'autre en Afrique du Nord, un troisième chez $V$. niloticus, qui habite les mêmes régions qu'exanthematicus, mais a une biologie différente, et une quatrième espèce chez $V$. exanthematicus.

En conclusion, l'analyse bibliographique, ainsi que les difficultés rencontrées au cours des essais de détermination, incitent à une 
grande prudence dans la systématique de ce groupe. La conception très uniciste des auteurs modernes nous parait excessive : si de nombreuses espèces paraissent effectivement peu spécifiques dans le choix de leur hôte, il semble bien qu'elles aient une répartition géographique beaucoup moins large que celle qui est habituellement admise. C'est pourquoi, alors que Morgan n'accepte que 24 espèces, nous croyons utile d'admettre l'individualité de 36 espèces.

La révision porte sur le groupe des Physaloptères pris au sens large, c'est-à-dire comprenant les trois genres : Skajabinoptera, Abbreviata et Physaloptera.

Tous les auteurs s'accordent sur la valeur systématique de la dentition céphalique. La crête denticulée qui orne la face interne des pseudo-lèvres varie légèrement d'un spécimen à l'autre dans un même lot, les éléments denticulés étant parfois plus petits et plus nombreux, ou plus grands et moins nombreux, mais ces variations ne semblent pas atteindre une grande amplitude. Les coupures systématiques que nous proposons portent donc essentiellement sur ces caractères céphaliques. L'anatomie génitale de la femelle, bien qu'elle puisse varier largement dans le détail, offre de bons éléments de différenciation si l'on ne s'appuie pas sur des éléments trop fins. La taille, et même jusqu'à un certain point la structure des spicules, peut également être utilisée. Enfin, la répartition des papilles cloacales et les caractères de l'ornementation ventrale de la queue du mâle, qui sont souvent considérés comme n'ayant aucune valeur, nous ont paru dans certains cas donner des éléments de diagnose parfaitement précis et valables. En revanche, la taille des spécimens, la longueur de l'œsophage, la situation de la vulve, les dimensions des œufs semblent tellement variables chez une même espèce que ces caractères ne sont utilisés que dans des cas exceptionnels.

Pour chercher à simplifier l'écriture, nous avons assigné artificiellement un

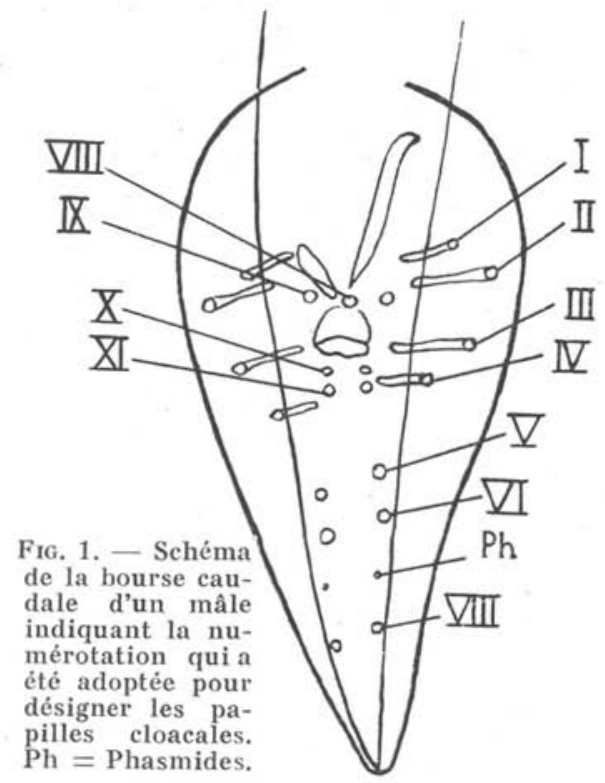


chiffre aux papilles cloacales. Elles sont numérotées de 1 à 7 d'avant en arrière sur le bord externe et de 8 à 11 autour du cloaque. 1, 2, 3 et 4 forment ainsi les quatre grandes paires pédonculées, 8 est la papille impaire précloacale, 9 la paire précloacale, et 10, 11 les deux paires accolées au bord postérieur du cloaque (fig. 1).

\section{TABLEAU DES ESPECES}

I. Pseudolèvre portant seulement une dent interno-latérale. Pas d'externo-latérale, ni de submédianes.

Genre Skrjabinoptera Schulz 1927

A Femelle avec deux utérus.

B Papilles 5 très antérieures, peu éloignées du niveau des papilles 4. Spicule gauche mesurant moins de $1 \mathrm{~mm}$. Parasite d'Iguanides nord-américains.

S. phrynosoma (Ortlepp 1922)

S. phrynosoma est bien connu grâce aux descriptions d'Ortlepp (1922), de Caballero (1937) et de Morgan (1942). C'est un parasite d'Amérique du Nord, du Mexique et non du Brésil (cf. Caballero). S. H. Lee (1955) a montré récemment, chez cette espèce, l'existence d'un très curieux mécanisme de ponte. L'espèce est souvent signalée chez différentes espèces d'Iguanes : Phrynosoma cornutum (Harlan), P. regale, $P$. douglasii (Bell.), P. solare Gray, P. plathyrhinos Girard, Sceloporus spinosus, S. torquatus, Anolis carolinensis Voigt.

B' Papilles 5 très postérieures au niveau des papilles 4 . Spicule gauche de $2 \mathrm{~mm}$. Parasite de Caméléons d'Afrique tropicale.

S. chamaeleontis (Gedoelst 1916)

Hôtes : Chamæleon gracilis (Hallowell) et Chamæleon sp., Congo belge.

A' Femelle avec quatre utérus.

C Espèce de grande taille (femelle longue de $44 \mathrm{~mm}$.). CEsophage relativement court. Parasite de Lézard australien.

S. simplicidens (Ortlepp 1922)

Hôte : "Sleeping Lizard », Australie. 
C' Espèce de petite taille (femelle longue de $8 \mathrm{~mm}$.). CEsophage relativement long. Parasite de Serpent européen (et non australien, comme il a été imprimé par erreur).

S. colubri (Rud. 1819)

Hôte : Coronella austriaca Laur. (= Zacholus austriaca), Autriche.

L'espèce est encore assez mal connue, car Ortlepp (1922) n'a eu que des femelles à sa disposition, et, actuellement, la meilleure description du mâle reste celle de Drasche (1883).

I'. Pseudolèvres portant plusieurs dents.

II. Pseudolèvre portant une dent externo-latérale, une dent interno-latérale et deux doubles dents submédianes.

Genre Abbreviata (Travassos 1920)

III. Pourtour buccal avec une denticulation homogène forte; la dent interno-latérale et les dents submédianes n'ont pas une taille nettement supérieure à celle des éléments de la crête denticulée et se confondent presque avec elle. Espèces généralement parasites d'Agames et de Geckos.

A Ornementation cuticulaire de la face ventrale de la queue du mâle limitée à une petite zone circulaire péricloacale.

B Les deux spicules sont petits (longueur inférieure à $400 \mu$ ).

C Naissance des utérus dichotomiques.

D Papilles cloacales 1 et 2 insérées à la même hauteur. Vulve antérieure à la fin de l'œsophage. Wufs grands $(65 \mu)$.

A. pallaryi (Seurat 1917)

Hôte : Agama bibroni A. Dumer., Maroc.

D' Papilles cloacales $1,2,3$ et 4 régulièrement étagées. Vulve postœsophagienne. Eufs petits $(45 \mu)$.

A. amaniensis (Sandground 1928)

Hôte : Agama mossambica Peters 1854, Tanganyika.

C' Les quatre utérus naissent au même point.

E Espèce de grande taille (femelle de 25-30 mm.). Ailes caudales du mâle remarquablement larges. Cufs petits $(50 \mu)$. Spicules non chitinisés.

A. achari (Mirza 1935) 
Hôte : Calotes versicolor (Daud.), Indes.

E' Espèce de petite taille (femelle de 7 à $12 \mathrm{~mm}$.). Ailes caudales du mâle normales. CEufs grands $(60 \mu)$. Spicules peu chitinisés, mais pourtant bien visibles.

A. baltazardi Chabaud 1953

Hôte: Phrynocephalus helioscopus (Pallas), Iran.

B' Les spicules sont très inégaux. Le gauche est long de 1,5 mm.

A. polydentata (Walton 1932)

Hôte : Hemidactylus mabouia (Moreau), Tanganyika.

A' L'ornementation cuticulaire de la face ventrale de la queue du mâle n'est pas limitée à une petite zone circulaire péricloacale.

F Ornementation cuticulaire de la queue du mâle occupant une zone arrondie passant au-dessous de la papille 6. Denticulation céphalique fine.

A. ortleppi (Sandground 1928)

Hôte : Chamæleo dilepis (Leach), Tanganyika.

F' Ornementation cuticulaire de la queue du mâle du type habituel, se prolongeant postérieurement en deux cornes le long des papilles 5 et 6 . Denticulation céphalique forte.

A. physignathi (Baylis 1924)

Hôte : Physignathus lesueurii (Gray), Australie.

III'. Les dents submédianes et la dent interno-latérale sont très nettement distinctes des éléments de la crête denticulée.

IV. Crête denticulée complète, occupant les angles ventral et dorsal de la bouche et l'espace compris entre les dents submédianes et les dents latérales.

A Les dents interno-latérales et submédianes sont modifiées et prennent un aspect papilliforme. Le bord libre des pseudolèvres est renforcé par un épaississement chitinoïde. Parasite de Tortues.

A. terrapenis (Hill 1941)

Hôte : Terrapene ornata (Agassiz), Amérique du Nord.

A' Dents interno-latérales et dents submédianes ayant l'aspect habituel. Parasite de Lacertiliens et d'Ophidiens. 
B Crête denticulée entre dents latérales et dents submédianes, comprenant seulement 3 ou 4 éléments de grande taille. Spicule gauche pas beaucoup plus grand que le droit, long de moins d'un millimètre.

A. antarctica (Linstow 1899) sensu Irwin-Smith, nec Ortlepp.

Hôtes : Acanthophis antarcticus Schaw., Tiliqua occipitalis $\mathrm{Pe}-$ ters, $T$. scincoides (Schaw.) (= Cyclodus boddaertii), T. nigrolutea Gray, Varanus varius (Schaw.), V. gouldi (Gray), Amphibolurus muricatus (Schaw.), Australie.

L'espèce décrite par Linstow chez deux Lézards australiens, $T$. occipitalis et $A$. antarcticus, est très probablement synonyme de $P$. alba Stossich, ainsi que l'indiquent Irwin-Smith et Ortlepp.

Le nom d'antarctica s'est trouvé être repris presque simultanément par Irwin-Smith et par Ortlepp, mais malheureusement pour deux espèces différentes. Il faut donc, ainsi que l'ont bien précisé Johnston et Mawson (1942 $a$ ), adopter la définition d'Irwin-Smith, car, d'une part, le travail a paru un peu antérieurement à celui d'Ortlepp, et, d'autre part, le matériel provient d'un Lézard zoologiquement très proche d'un de ceux dont parle Linstow. L'espèce décrite sous le même nom par Ortlepp a été reprise par Johnston et Mawson sous le nom de $A$. confusa.

A. antarctica a été retrouvé à différentes reprises par Johnston et Mawson (1943, 1947, 1951) chez des Lézards et non des Serpents. Irwin-Smith a décrit une variété lata dont le corps de la femelle est élargi postérieurement et dont l'œsophage est un peu plus long. L'hôte n'est pas connu avec certitude, mais pourrait être une autre espèce de Lézard australien : Egernia cunninghami.

B' Crête denticulée entre dents latérales et dents submédianes comprenant plus de 3 ou 4 éléments. Spicule gauche, beaucoup plus grand que le droit, long de plus d'un millimètre.

C Spicule droit, long de $500 \mu$. Espèce australienne.

A. gallardi (Johnston et Mawson 1942 b)

Hôtes: Amphibolorus barbatus (Cuv.) et A. muricatus (Schaw.). (Cf. J. et M., 1943). 
C’ Spicule droit, ne dépassant pas $400 \mu$. Espèces paléarctiques ou éthiopiennes.

D Dent latérale externe tronquée à l'apex. Parasite de Reptiles paléarctiques.

E Trompe impaire assez longue, dichotomiquement divisée en 4 branches. Spicule droit, long d'environ $180 \mu$.

A. abbreviata (Rud. 1819)

Hôtes : L'espèce décrite chez «Lacerta margaritacea 》 (1), en Espagne, par Rudolphi, a été reprise avec les bonnes descriptions de Seurat $(1914,1917)$ pour un matériel provenant de L. lepida d'Algérie. Le nom a été employé par de nombreux auteurs pour des spécimens variés, et la liste des hôtes est difficile à établir, car beaucoup de déterminations sont manifestement inexactes.

Le Rongeur Graphiurus murinus, cité par Parona (1909), ainsi que le Ouistiti brésilien Jacchus vulgaris, mentionné par Stiles et Hassall (1920), sont évidemment des erreurs. Diesing (1851), ayant établi la synonymie avec l'Ascaris fallax de Rudolphi, donne comme hôtes : L. viridis (Laur.) et $L$. lepida en Espagne. Les références de Leidy concernant les Iguanes américains Phrynosoma douglasii hernandesi Girard et $P$. regale proviennent d'erreurs de détermination, ainsi qu'a pu le montrer Walton (1927). Linstow (1883) donne le même nom d'abbreviata à l'ensemble d'un matériel récolté au Turkestan chez Ophisaurus apodus (Pallas) (= Pseudopus pallasii), Natrix tesselatus (Laur.) (= Tropidonotus hydrus), (?) Phrynocephalus helioscopus (Pallas), et comme pseudo-parasite Ciconia alba. Si la description de l'auteur est exacte, la denticulation labiale, la taille des œufs et la longueur de l'œsophage s'opposent nettement à l'espèce de Seurat. Il est donc bien difficile de savoir quelle est l'espèce qui a été étudiée par Linstow. Skrjabin (1916) détermine également comme $P$. abbreviata des femelles immatures récoltées chez Agama sp. en Afrique orientale. Le seul élément précis donné par la denticulation céphalique ne correspond pas à la description de Seurat. Lopez-Neyra (1947) a identifié l'espèce en Espagne chez L. lepida (= Gongylus ocellatus) et Lacerta muralis (Laur.).

(1) Lacerta lepida Daudin $=L$. ocellata $=L$. margaritata Schinz, $1833=$ probablement L. margaritacea. 
Stiles et Hassall (1920) citent également L. agilis L., L. muralis (Laur.) et L. vivipara Jacquin.

Il apparait donc, en conclusion, que toutes les références autres que celles qui concernent les Lézards d'Espagne et d'Afrique du Nord sont fausses ou suspectes; nous croyons que la répartition géographique de l'espèce est strictement paléarctique et qu'elle est peut-être même en réalité limitée à la région méditerranéenne.

E' Trompe impaire courte, immédiatement divisée en 4 branches. Spicule droit long d'environ $100 \mu$.

A. algeriensis nom. nov.

(= paradoxa, sensu Seurat 1914 ;

$=$ A. varani, sensu Seurat 1917).

Hôtes : Varanus griseus Daudin ; Chamæleo vulgaris Daudin ; Aspis cerastes $($ L.) $(=$ Cerastes cornutus $)$, Algérie.

Seurat, en 1914, a identifié son espèce à l'A. paradoxa décrit par Linstow en 1908 chez un Varan d'Afrique du Sud, puis, en 1917, il admet la synonymie avec varani décrit par Parona chez un Varan asiatique; il désigne donc son espèce sous le nom de varani. Ortlepp, en 1922, ayant eu l'avantage d'avoir un matériel asiatique (correspondant au même hôte que celui de Parona) et un matériel africain (correspondant au même hôte que celui de Linstow), donne des caractères différentiels précis entre les deux espèces. D'après ces caractères, l'espèce de Seurat ne peut être rapportée à varani, mais plutôt à paradoxa (cadre buccal denticulé, utérus naissant tous au même point).

Ortlepp constate quelques différences entre son matériel et la description de Seurat, mais admet pourtant la synonymie : « Despite these differences, I believe the parasite to be the same, and the difference in size of the spicules can be accounted for in that it is not always possible to measure them correctly, as they generally take up a twisted course inside the body. \$ Les auteurs qui ont suivi, et en particulier Morgan (1945), admettent ce point de vue. Une erreur de $80 \mu$ sur la mensuration d'un spicule mesurant $100 \mu$ parait bien improbable dans un texte de Seurat. En outre, les spécimens d'Ortlepp ont une dent latérale aiguë, alors que Seurat précise : "Dent labiale externe cunéiforme, nettement tronquée 
à l'extrémité libre ». Nous sommes donc persuadé que l'espèce de Seurat est différente de celle d'Ortlepp, et nous croyons devoir la désigner sous le nom nouveau d'A. algeriensis.

D' Dent latérale externe aiguë à l'apex. Parasites de Reptiles éthiopiens.

F Trompe impaire immédiatement divisée en 4 branches.

A. paradoxa (Linst. 1908) sensu Ortlepp 1922

Nous avons vu plus haut (discussion sur A. algeriensis) que l'espèce a pu être individualisée grâce aux travaux d'Ortlepp (1922), qui a décrit son matériel à partir de l'hôte type : Varanus albigularis (Daud.), d'Afrique du Sud.

Monnig (1924), qui n'a pas encore connaissance du travail d'Ortlepp, assimile, comme Seurat, le Physaloptère d'Afrique du Sud à varani, bien que les lèvres soient denticulées et que les 4 branches utérines naissent au même point. En 1934, Mirza donne une description d'une espèce denticulée, intermédiaire entre varani et paradoxa, qui serait adulte chez un Varan et chez un Ecureuil. Nous croyons, comme Baylis (1939), " that two species are here inextricably confused », et qu'il est impossible de tenir compte de cette référence.

Il semble ainsi qu'il n'y ait dans la littérature aucune référence valable à $A$. paradoxa en dehors de celles de Linstow, Ortlepp et Monnig, et que les seuls hôtes connus soient V. albigularis d'Afrique du Sud, Psammophis sibilans (L.) du Soudan et d'autres Serpents indéterminés de Nigéria et du Soudan. Cependant, en 1939, Baylis, après avoir indiqué que paradoxa n'existait pas aux Indes, indique dans une note infrapaginale: "Since this was written, specimens from Varanus monitor (L.) in Hyderabad, have been determined as $P$. paradoxa. \& Cette brève indication montre bien la présence d'une espèce très proche de paradoxa en Asie, mais, d'après ce que nous voyons sur la répartition géographique des autres espèces, il semble qu'une étude comparée des spécimens serait nécessaire pour pouvoir affirmer la similitude des deux formes.

F' Trompe impaire dichotomiquement divisée en 4 branches.

A. bayfisi nom. nov. (=P. quadrovaria sensu Baylis et Daubney 1923 ; $=P$. paradoxa sensu Kung 1948). 
Hôte: Varanus exanthematicus (L.), Afrique équatoriale.

Quelques spécimens en mauvais état, provenant de Varanus exanthematicus de Nigéria, ont été déterminés avec hésitation par Baylis et Daubney (1923) comme P. quadrovaria. Ces auteurs constatent en effet que la structure génitale est plus proche de celle de varani que de celle de quadrovaria; mais la structure céphalique leur semble avoir plus de valeur, et ils constatent l'existence d'une crête denticulée qui élimine varani.

En 1948, C. C. Kung décrit très brièvement chez un V. exanthematicus, dont la provenance n'est pas indiquée, un Physaloptère qu'il détermine comme $P$. paradoxa, mais où il remarque une division dichotomique de l'utérus.

Ayant étudié une femelle récoltée chez le même hôte au Congo (la description en sera donnée ultérieurement en collaboration avec R. Rousselot), nous avons constaté le même phénomène. La structure céphalique répond à la description du paradoxa d'Ortlepp, mais la division des utérus est nettement dichotomique.

Nous voyons donc que tous les spécimens récoltés à différentes reprises chez le même hôte $V$. exanthematicus ont des caractères constants qui les opposent aux espèces voisines, et nous croyons qu'il est utile de les désigner sous le nom nouveau d'A. baylisi.

IV'. Crête denticulée incomplète ou absente.

V. Crête denticulée présente entre les dents submédianes et la dent latérale, absente dans les angles dorsal et ventral de la bouche.

A Femelle avec deux utérus.

B Spicules peu inégaux. Ornementation caudale du mâle limitée à un cercle péricloacal.

A. gracilis (Ortlepp 1922)

Hôte : Lézard indéterminé d'Ouganda. Cette espèce a des affinités avec les Abbreviata du premier groupe, parasites d'Agames et de Geckos.

B' Spicules très inégaux. Ornementation caudale du mâle du type normal, largement étendue sur une aire cordiforme.

A. leptosoma (Gervais 1848) 
Hôtes : Uromastix acanthinurus Bell. ; Varanus griseus Daud., Algérie.

L'espèce a été récoltée en abondance à l'Institut de Parasitologie, dans les fosses nasales d'un $U$. acanthinurus provenant de Biskra. Le matériel correspond parfaitement à la description de Seurat. La denticulation céphalique, bien que très réduite, semble constante; le nombre des éléments entre une dent submédiane et la dent latérale varie de 3 à 8 .

La synonymie de A. leptosoma et de S. chamæleontis Gedoelst 1916, admise par Irwin-Smith et par Ortlepp, a été rejetée par Morgan ; les deux espèces ont beaucoup d'affinités, mais les hôtes et la région géographique sont différents. Les dents submédianes, très apparentes chez leptosoma, sont absentes chez chamæleontis.

A' Femelle avec quatre utérus.

C Crête denticulée peu marquée. Spicules grands et très inégaux ; la pointe du spicule gauche en forme de spatule. Parasite de Serpents et de Lézards australiens. Division de l'utérus fortement dichotomique.

A. confusa (Johnston et Mawson 1942) (=A. antarctica sensu Ortlepp nec Irwin-Smith).

Hôtes : L'espèce décrite en 1922 chez Varanus varius Shaw et Python spilotes Lacep. a été retrouvée très fréquemment par Johnston et Mawson (1946, 1948, 1951) chez de nombreux Reptiles australiens: Notechis scutatus Peters, Demansia textilis Dum. et Bibr., D. psammophis Schl., D. reticulata Gray, Pseudechis porphyriacus Shaw, P. australis Gray, Acanthophis antarctica Shaw, Denisonia superba Gunth., D. suta Peters, Varanus gouldi Gray, $\boldsymbol{V}$. belli D. et B., et $\boldsymbol{V}$. giganteus Gray.

C' Crête denticulée bien marquée. Spicules petits et peu inégaux. Division de l'utérus non dichotomique. Parasite de (?) Chamæsaura en Rhodésie.

\section{A. tasmani (Ortlepp 1937)}

Hôte : (?) Chamæsaura macrolepis Cope $[=(?)$ Chameleon macrolepis], Rhodésie méridionale.

Le nom de «Chameleon macrolepis » donné par Ortlepp ne figure pas dans les catalogues de Reptiles que nous avons pu 
consulter. M. J. Guibet suppose qu'il s'agit en réalité d'un Chamæsaura, Reptile proche des Anguidæ, et n'ayant aucun rapport avec les Caméléons.

C" Crête denticulée bien marquée. Division des utérus faiblement dichotomique. Mâle inconnu. Parasite de Varans africains.

A. quadrovaria (Leiper 1908)

Hôte : Varanus niloticus (L.), Soudan.

L'espèce n'est connue que par la femelle, mais Ortlepp (1922) a donné des caractères précis pour la différencier des formes voisines, et en particulier de paradoxa et de varani. Il a maintenu ces caractères différentiels dans le tableau dichotomique de 1937. Morgan (1941, et travaux ultérieurs) place l'espèce en synonymie de varani, sans préciser la raison qui l'a déterminé à cette décision. Nous ne pouvons donc pas suivre cet auteur, et considérons l'espèce comme valide, d'autant plus que l'hôte Varanus niloticus a des mœurs aquatiques qui l'éloignent écologiquement des autres Varans de la même région.

V'. Crête denticulée absente entre les dents submédianes et la dent latérale.

VI. Crête denticulée présente dans les angles ventral et dorsal de la bouche.

A Mâle long de 10,5 mm. ; femelle de $30 \mathrm{~mm}$. ; œufs de $57 \mu$.

A. bancrofti (Irwin-Smith 1922)

Hôte : Varanus varius Shaw, Australie.

A' Mâle long de 6-8 mm. ; femelle de 10-14 mm.; œufs de 26$48 \mu$.

A. oligopapillata (Kreis 1940)

Hôte : Sphenomorphus jobiensis (Meyer), Océanie.

Kreis n'a pas donné de caractères différentiels avec les autres Physaloptères, et les mensurations coïncident assez bien avec celles que donne Irwin-Smith pour bancrofti. Les différences proposées ici ne suffisent pas pour différencier sûrement les deux espèces et ne sont données qu'à titre provisoire.

VI'. Bouche n'ayant pas ces caractères. 
VII. Crête denticulée complètement absente.

A Papille caudale 7 très longuement pédonculée.

A. demansiae (J. et M. 1948)

Hôte : Demansia psammophis (Schl.), Australie.

Les auteurs se sont basés sur les papilles cloacales du mâle pour différencier leur espèce, et la structure céphalique n'est décrite et figurée que très succinctement. Il semble pourtant qu'on puisse classer l'espèce dans le groupe des Abbreviata dépourvus de crête denticulée.

A' Papille caudale 7 normale, très faiblement pédonculée.

B Papilles caudales 1-2 et 3-4 formant deux groupes largement espacés. Parasite de Serpents africains.

A affinis (Gedoelst 1916)

Hôtes: Psammophis sibilans (L.), Crotaphopeltis hotamboeia Laur., Afrique équatoriale.

L'espèce a été mise en synonymie de paradoxa par Ortlepp, car cet auteur, ayant étudié un Physaloptère du même hôte, l'a identifié à paradoxa.

La synonymie a été admise par les auteurs postérieurs à Ortlepp, bien que, dans la description de Gedoelst, on trouve des éléments qui l'opposent nettement à paradoxa. En outre, Baylis (1939), ayant examiné le matériel original de Gedoelst, « finds that it is quite distinct from the form described by Ortlepp as $P$. paradoxa ». Enfin, en 1940, Baylis admet la validité de l'espèce.

Un Abbreviata, parasite de Crotaphopeltis hotamboeia, récolté par R. Rousselot à Brazzaville (dont nous publierons la description ultérieurement), présente les caractères décrits par Gedoelst : absence de crête denticulée, répartition des quatre premières papilles cloacales en deux groupes distincts.

L'espèce doit donc être séparée nettement de paradoxa et le nom proposé par Gedoelst est valable.

B' Les quatre premières paires de papilles cloacales sont régulièrement espacées.

C Cloaque entouré de verrues proéminentes. Spicule gauche long d'environ $500 \mu$.

A. leidyi (Walton 1927) 
Hôte: Varanus varius (Schaw) $=$ V. varians (Australie).

C' Le cloaque n'est pas entouré de verrues proéminentes. Spicule gauche long de plus d'un millimètre.

D Spicules relativement petits (droit 235-261 $\mu$, gauche 1,34$1,46 \mathrm{~mm}$.). Parasite de Serpents de la région australienne.

A. natricis (Kreis 1940)

Hôte : Natrix hypomelas Günther.

L'espèce a été mise en synonymie de bancrofti et de physignathi par Morgan; mais les descriptions des structures céphaliques sont nettement différentes. L'espèce nous parait plus difficile à différencier morphologiquement de varani. La diagnose n'a pas été précisée par l'auteur et la différence dans la taille des spicules que nous donnons à titre provisoire dans le tableau dichotomique est trop faible pour avoir une grande valeur.

D' Spicules relativement grands (droit 306-374 $\mu$, gauche 1,83 à 2,20 mm.). Parasite de Reptiles asiatiques et (?) américains.

A. varani (Parona 1889) sensu Ortlepp 1922.

L'espèce, dont l'hôte type est Varanus monitor à Ceylan, a été reprise par Ortlepp sur un matériel provenant du même hôte de Ceylan et de Varanus indicus de l'Inde. Baylis (1939) souligne les nombreuses différences entre les descriptions des deux auteurs, et pense que deux espèces distinctes sont peut-être en cause. Tant qu'une autre espèce plus conforme à la description de Parona n’aura pas été décrite, nous croyons qu'il y a tout avantage à conserver la détermination d'Ortlepp. La redescription récente de Cruz (1950) correspond bien à celle d'Ortlepp.

Hsü et Hoeppli (1931) ont décrit sous le même nom un Abbreviata parasite d'un Serpent de Chine. L'espèce est morphologiquement très proche de varani, mais les auteurs signalent cependant un certain nombre de différences, et nous partageons l'avis de Baylis qui considère l'identification spécifique avec varani comme douteuse.

Plusieurs auteurs compétents ont identifié à varani un Abbreviata parasite de divers Reptiles américains. Pourtant, étant donné, d'une part, la grande analogie morphologique des différentes espèces d'Abbreviata, et, d'autre part, l'étroite ré- 
partition géographique de toutes les autres espèces parasites de Reptiles, on peut se demander si cette identification ne doit pas être remise en question, et s'il n'y a pas deux formes extrêmement proches actuellement confondues sous le même nom.

II'. Bouche n'ayant pas ces caractères.

VIII. Pseudolèvres portant une dent externo-latérale et un groupe interno-latéral de trois dents. Dents submédianes absentes.

Genre Physaloptera Rud. 1819

A Utérus naissant dès la chambre à œufs.

B Spicules longs de $455 \mu$.

P. bonnei Ortlepp 1922

Hôte : «Sapakara », Guyane hollandaise.

L'hôte n'est connu que par son nom local et sa place zoologique reste inconnue. Il est probable cependant, ainsi que le pense Morgan (1943), qu'il s'agit d'un Reptile, car l'aspect en lancette du spicule gauche ne s'observe pas chez les Physaloptères d'Oiseaux et de Mammifères.

B' Spicules longs de $285 \mu$.

P. abjecta Leidy 1856

$(=P$. variegata Reiber, Byrd et Parker 1940)

Hôtes : Masticophis flagellum (Shaw), C. constrictor (L.), Lampropeltis getulus (L.), Thamnophis sirtalis (L.), Liopeltis vernalis (Harlan), Etats-Unis.

L'espèce, bien décrite par Reiber et ses collaborateurs (1940) sous le nom de $P$. variegata, a été utilisée par Morgan (1943) sous le nom de P. abjecta Leidy. Nous suivrons ici cette décision, bien qu'elle puisse paraitre un peu arbitraire, car elle élimine une sp. inquirenda.

A' Utérus unis à la chambre à œufs par l'intermédiaire d'un tronc commun.

C Spicule droit fortement courbé en hameçon.

P. retusa Rud. 1819

(=P. mucronata Leidy 1856 ;

$=P$. lagarda Sprehn 1932). 
Hôtes : Nombreux Téjidés et Ignanidés au Brésil, Bolivie, Guyane hollandaise (Baylis, 1947) et Etats-Unis.

L'identification du parasite en Nouvelle-Bretagne chez Boiga irregularis (Merren) (= Dipsadomorphus irregularis) donnée par Shipley (1899) a besoin d'être vérifiée.

Walton (1927), ayant étudié le $P$. mucronata de Leidy, a pu le mettre en synonymie. Sprehn (1932) décrit comme espèce nouvelle $(P$. lagarda) un mâle provenant de Tupinambis rufescens (Günth), qui a tous les caractères de retusa, mais qui est un peu plus grand que les spécimens d'Ortlepp. Ceux de Walton atteignent des dimensions comparables à l'exemplaire de Sprehn, et la mise en synonymie faite par Morgan semble justifiée.

C' Spicule droit faiblement courbé ventralement.

D Espèce de petite taille (mâle long de moins de $15 \mathrm{~mm}$., femelle de moins de $20 \mathrm{~mm}$.). Dents latérales internes très petites. Vulve très postérieure à la fin de l'œsophage.

P. squamatae Harwood 1932

Hôtes : Leiolopisma laterale (Say), Agkistrodon mokasen Beauvois, Texas.

Les différences entre les descriptions de Harwood et d'Ortlepp pour $P$. obtusissima paraissent trop considérables pour que l'on puisse admettre a priori l'identification donnée par Morgan.

D' Espèce de grande taille (mâle long de plus de $15 \mathrm{~mm}$., femelle de plus de $20 \mathrm{~mm}$.). Dents latérales internes plus petites que l'externe. Vulve un peu en arrière de la fin de l'œsophage.

\section{P. monodens Molin 1860}

Hôte : Constrictor constrictor (L.), Brésil.

L'espèce a été redécrite par Ortlepp (1922) à partir du matériel original, et différenciée de l'espèce suivante, $P$. obtusissima, par la taille des dents, la place de la vulve et la longueur de l'œsophage.

Nous ne croyons donc pas devoir retenir la mise en synonymie donnée sans explications par Morgan puisque, contrairement à Ortlepp, l'auteur n'a pu comparer les deux espèces.

D” Espèce de grande taille (mâle long de plus de $15 \mathrm{~mm}$., femelle 
de plus de $20 \mathrm{~mm}$.). Dents latérales internes aussi grandes que l'externe. Vulve très postérieure à la fin de l'œsophage.

P. obtusissima Molin 1860

Hôtes : Nombreux Serpents du Brésil et des Etats-Unis.

Il serait utile de revoir les spécimens de Nouvelle-Bretagne déterminés sous le même nom par Stossich (cf. Shipley, 1899).

\section{Espèces mal connues.}

A cette longue liste d'espèces, il faut ajouter une série de sp. inquirendæ qui ne peuvent figurer dans le tableau, soit parce que la description est insuffisante, soit parce que le seul sexe connu ne permet pas une diagnose complète avec les autres espèces. Nous chercherons à les classer suivant les groupes utilisés plus haut.

Groupe I : S. sonsinoi (Linst. 1895), parasite du Lézard égyptien Agama mutabilis Merrem, est classé parmi les Abbreviata. Cependant, l'ornementation cuticulaire de la queue du mâle n'a pas le type habituel aux Abbreviata parasites d'Agames, et Linstow ne parle que d'une seule dent céphalique. Les Physaloptères de Reptiles au sens strict (groupe VIII) paraissent strictement limités au Nouveau-Monde. Nous supposons done que l'espèce est un Skrjabinoptera.

Groupe III : A. britanica (Skrjabin 1916) chez Agama sp. (Afrique orientale) ; A. aloisii-sabaudiæ (Parona 1907) chez Agama atricollis Smith (Afrique); A. dentata (Linst. 1883) chez Agama sanguinolente, Vipera beras (L.) et Phrynocephalus mystaceus (Pallas) (= Phrynocephalus auritus) (Turkestan).

Groupe IV : A. clelandi (Irwin-Smith 1922) chez Varanus varius (Australie). Le mâle reste inconnu.

Groupe V : A. heterocephala, femelle (Kreis, 1940), chez Gonyocephalus modestus (Meyer), en Nouvelle-Bretagne. Kreis a décrit une espèce dont le mâle et la femelle auraient une structure céphalique très différente. Ces cas de dimorphisme céphalique sont connus chez quelques rares Oxyures ou Tétramères, dans des fa. milles où le dimorphisme sexuel est très marqué, mais ils paraissent bien peu vraisemblables dans le cas des Physaloptères. Les dimensions données par Kreis ne portant que sur un seul spécimen de chaque sexe, il semble bien que l'auteur n'ait eu qu'un seul mâle et qu'une seule femelle, et nous sommes persuadé qu'il s'agit de deux espèces différentes. 
Groupe VI : A. heterocephala, mâle (Kreis, 1940); A. spiralis (Schneider 1866) chez Amphisbæna sp. (Brésil).

\section{REMERCIEMENTS}

M. Jean Guibet, du Muséum d'Histoire Naturelle de Paris, a bien voulu réviser la nomenclature des Reptiles et nous indiquer les synonymies nécessaires. Nous l'en remercions très sincèrement.

\section{RÉSUMÉ}

Les difficultés rencontrées au cours de la détermination des Physaloptères de Reptiles, ainsi que l'analyse critique de la bibliographie, nous ont conduit, dans ce groupe, à admettre une systématique beaucoup moins uniciste que celle qui apparaît dans les récentes publications faites à ce sujet. Il ne semble pas que les espèces aient la très vaste extension géographique qui leur est habituellement attribuée, et, contrairement à ce que l'on observe habituellement chez les Nématodes parasites, la répartition des espèces nous semble être déterminée aussi bien par la répartition géographique que par la nature zoologique de l'hôte.

Alors que Morgan accepte seulement 24 espèces, nous proposons d'admettre la validité de 36 espèces.

Nous avons cherché à construire un tableau dichotomique basé essentiellement sur les caractères céphaliques, et les espèces se trouvent ainsi scindées en sept groupes :
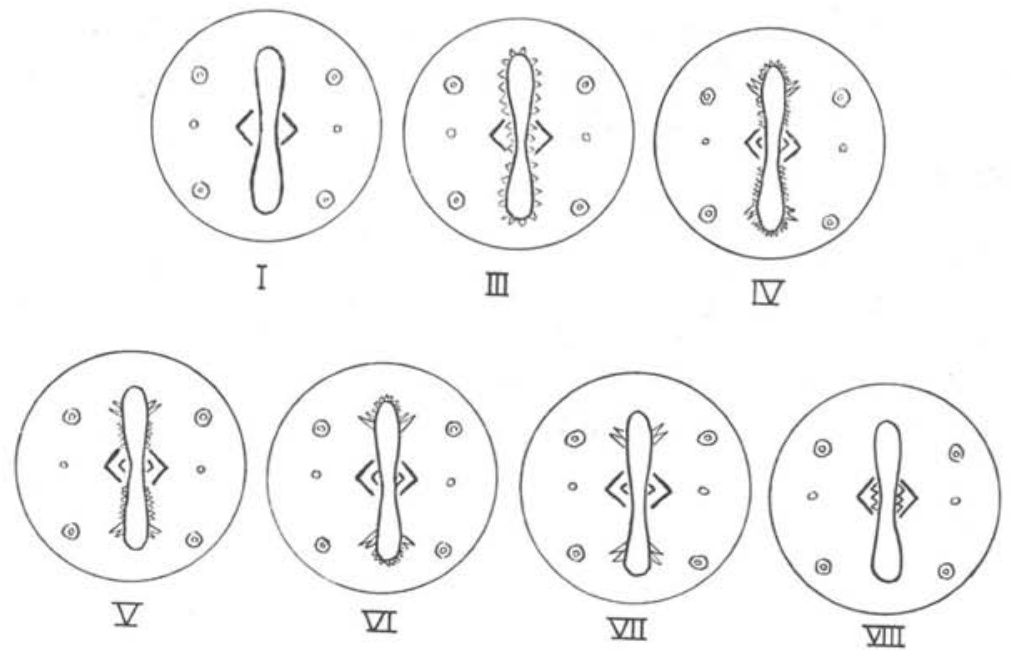

FIG. 2. - Différents types de denticulation céphalique. Groupe I. Ex. : S. phrynosoma. $\rightarrow$ Groupe III. Ex. : A. baltazardi. - Groupe IV. Ex. : A. abbreviata. - Groupe V. Ex. : A. leptosoma. - Groupe VI. Ex. : A. bancrofti. Groupe VII. Ex. : A. varani. - Groupe VIII. Ex. : P. retusa. 
I. Skrjabinoptera : une seule dent interno-latérale.

II. Abbreviata : une dent externo-latérale, une dent interno-latérale et deux doubles dents submédianes.

Le groupe est scindé en cinq groupes :

III. Denticulation homogène ; la dent interno-latérale et les dents submédianes sont confondues avec la crête denticulée.

IV. Crête denticulée complète, mais nettement distincte de la dent interno-latérale et des dents submédianes.

V. Crête denticulée présente seulement entre les dents submédianes et la dent latérale.

VI. Crête denticulée présente seulement dans les angles ventral et dorsal de la bouche.

VII. Crête denticulée absente.

VIII. Physaloptera : une dent externo-latèrale, un groupe internolatéral de trois dents; dents submédianes absentes.

A côté de nombreuses espèces précédemment mises en synonymie, et dont nous admettons ici la validité, apparaissent deux noms nouveaux :

A. algeriensis $(=P$. paradoxa sensu Seurat $1914 ;=P$. varani sensu Seurat 1917), et

A. baylisi (=P. quadrovaria sensu Baylis et Daubney 1923 ; $=P$. paradoxa sensu Kung 1948). 


\section{Bibliographie}

Baylis (H.-A.), 1924. - A new species of Physaloptera (Nematoda) from an Australian Lizard. Ann. Mag. Nat. Hist., Ser. 9, XIII, 309-311, fig. A-B.

BAyLIS (H-A.), 1939. - The Fauna of British India including Ceylon and Burma. Nematoda, vol. II (Filarioidea, Dioctophymoidea and Trichinelloidea), Londres, xxvin + 274 pp., fig. 1-150.

Bayles (H. A.), 1940. - On a further Collection of Parasitic Worms from the Belgian Congo. Ann. Mag. Nat. Hist., (Ser. 11), V, 401-417, fig. 1-6.

Baylis (H.-A.), 1947. - Some roundworms and flatworms from the West Indies and Surinam. L. Nematodes and Acanthocephala. Lin. Soc. J. Zool., XLI $(n \circ 280)$.

BAylis (H.-A.) et DAUBNey (R.), 1923. - A further report on parasitic nematodes in collection of the Zoological Survey of India. Rec. of the Indian Mus., $\mathrm{XXV}$, VI, 551-578.

Ciaballero (E. y C.), 1937. - Nematodes de algunos vertebrados del valle del Mezquital, Hgo. Anal. Inst. Biol. Mexico, VIII, 189-200, fig. 1-6.

Chabaud (A.-G.), 1953. - Un nouveau Physaloptère parasite d'Agame. Ann. Parasit., XXVIII, 305-311, fig. 1-2.

Cruz (H.), 1950. - Observations on some Spirurid Nematodes from Ceylon Vertebrates. Ceylon J. Sc. (B.), XXIV, pr. 2.

Diesing (C. M.), 1851. - Systema helminthum, vol. II, Vindobonae, vi + 591 pp.

Drasche (R. von), 1883. - Revision der in der Nematoden-Sammlung des k. k. Zoologischen Hofeabinetes befindlichen Original Exemplare Diesing's und Molin's. Verhandl. d. k. k. zool. bot. Gesellsch. in Wien (1882), XXXII, 117-218.

Gedoerst (L.), 1916. - Notes sur la Faune Parasitaire du Congo Belge. Rev. Zool. Africaine, V, 1-90, fig. 1-20.

HARwood (P. D.), 1932. - The Helminths parasitic in the Amphibia and Reptilia of Houston, Texas and vicinity. Proc. U.S. Nat. Mus., 81, 17, 1-71, pl. 1-5.

Hils. (W.), 1940. - The genus Physaloptera Rud. Wasmann Collector, IV, 60-70. (Cet ouvrage n'a pas été consulté).

Hul. (W. C.), 1941. - Physaloptera terrapenis, a new Nematode from a Tortoise. Trans. Amer. Microsc. Soc., LX, 59-64, fig. 1-8.

Hsü (H. F.) et Hoeppli (R.), 1931. - Parasitic Nematodes mostly from smakes collected in China. The Nat. Med. J. of China, XVII, 567-588.

Inwiv-Sмiтн (V. A.), 1922. - Notes on Nematodes of the genus Physaloptera, with special reference to those parasitic in Reptiles. The Proc. of the Linn. Soc. of N. S. W., XLVI, 1921, 492-502, XLVII, 53-62, fig. 1, 232244, fig. 1-38, 415-427, fig. 1-38.

Ans. de Parastrologie, T. XXXI, $x^{\circ} 1-2-1956$. 
Johnston (T. H.) et MAwson (P. M.), $1942 a$. - Some new and known australian parasitic Nematodes. The Proc. of the Linn. Soc. of N.S. W., LXVII, 90-94, fig. 1-12.

Johnston (T. H.) et Nifwson (P. M.), $1942 b$. - The Gallard collection of parasitic nematodes in the australian Museum. Rec. of the Aust. Mus., XXI, 110-115, fig. 1-18.

Johnston (T. H.) et Mawson (P. M.), 1943. - Remarks on some Nematodes from australian Reptiles. Trans. Roy. Soc. Sth. Aust., LXVII, 183-186, fig. 1-5.

Johnston (T. H.) et Mawson (P. M.), 1947. - Some Nematodes from australian Lizards. Trans. Roy. Soc. Sth. Aust., LXXI, 22-27, fig. 1-13.

Johnston (T. H.) et Mawson (P. M.), 1948. - Some new records of Nematodes from australian snakes. Rec. of the Sth. Aust. Mus., IX, 101-106, fig. 1-8.

Johsston (T. H.) et Mawson (P. M.), 1951. - Report on some parasitic Nematodes from the australian Museum. Rec. of the Aust. Mus., XXII, 289297, fig. 1-7.

KreIs (H. A.), 1940. - Beiträge zur Kenntnis parasitischer Nematoden, IX. Parasitische Nematoden aus dem Naturhistorischen Museum Basel. Zentr. für Bakterio. Parasit. und Infek., Orig. 1 Abt., CXLV, 163-208, fig. 1-17.

Kung (C. C.), 1948. - On some species of Spirurids from terrestrial Vertebrates, with notes on Habronema mansioni, Physaloptera paradoxa and Hartertia zuluensis. Jl. Helminth., XXII, 141-164, fig. 1-40.

LeE (S. H.), 1955. - The mode of egg dispersal in Physaloptera phrynosoma Ortlepp (Nematoda : Spiruroidea), a gastric Nematode of Texas Horned Toads, Phrynosoma cornutum. J. Parasit., XLI, 70-74, fig. 1-3.

LEIPER (R. T.), 1908. - An account of some Helminthes contained in Dr. Wenyon's collection from the Sudan. Rept. Wellcome Res. Labor., III, 187-194.

Linstow (O. von), 1883. - Nematoden, Trematoden und Acanthocephalen gesammelt von Prof. Fedtschenko in Turkestan. Arch. f. Naturgesch., XCVIII, 274-314 + pl. VI-IX.

Lisstow (O. von), 1895. - Untersuchungen an Nematoden. Arch, f. mikr. Anat., XLIV, $509-533+$ pl. xxx-xxxI.

Lisstow (O. von), 1908. - Nematoden und Acanthocephalen aus dem westlichen und zentralen Südafrika. Denkschriften der mediz. naturwis. Gesell., Bd. XIII, 21-28 + pl. w.

Lopez-Neyra, (C. R.), 1947. - Helmintos de los Vertebrados ibéricos, Grenade, 1.212 pp., pl. 1-174.

Mirza (M. B.), 1934. - Sciurus palmarum als ein interessanter Wirt von Physaloptera sp. Zeitsch. f. Parasit., VI, 638-641, fig. 1-4.

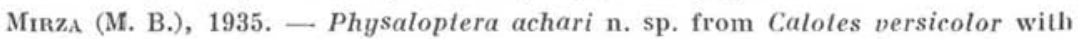
a short note on abnormalities in the genus Physaloptera. Proc. Acad. Sc., U.P., India, V, 71-74, fig. 1-4.

Molin (R.), 1860. - Una monographia del genere Physaloptera. Sitzungsh. Akad. Wissensch., Vienne, XXXIX, 637-672. 
Monnig (H. O.), 1923. - South African Parasitic Nematodes. 9th and 10th Rep. Direct. Veter. Educ, and Research, Onderstepoort, 435-478, fig. 1-46.

Morgan (B. B.), 1941. - A summary of the Physalopterine (Nematoda) of North America. Proc. of the Helm. Soc. of Washington, VIII, 28-30.

Morgan (B. B.), 1942. - The Nematode genus Skrjabinoptera Schulz 1927. Lloydia, V, 314-319.

Morgan (B. B.), 1943. - The Physaloptera (Nematoda) of Reptiles. Le naturaliste canadien, LXIX, 179-185.

Morgan (B. B.), 1945. - The Nematode genus Abbreviata (Travassos 1920), Schulz, 1927. The Amer. Midland. Natural., XXXIV, 485-490.

Morgan (B. B.), 1946. - Host-parasite relationships and geographical distribution of the Physalopteride (Nematoda). Trans, of the Wiscon. Acad. Sc., XXXVIII, 273-292, fig. 1-16.

Ortlepp (R. J.), 1922. - The Nematode genus Physaloptera Rud. Proc. Zool. Soc. Lond., 999-1107, fig. 1-44.

Ortlepp (R. J.), 1937. - Some undescribed species of the nematode genus Physaloptera Rud., together with a key to the sufficiently known forms. Onderstep. Jl. of Veter. a. Anim. Ind., IX, 71-84, fig. 1-8.

РавоNA (C.), 1909. - Vermi parassiti di vertebrati. Ruwenzori, Milano, parte scient., vol. 1, 415-422, fig. 1-5.

Reiber (R. J.), Byrd (E. E.) et Parker (M. V.), 1940. - Certain new and already known Nematodes from Amphibia and Reptilia, Lloyda, III, 125-144.

Rudolphi (C. A.), 1819, - Entozoorum synopsis, cui accedunt mantissa duplex et indices locupletissimi. Berolini, $+811 \mathrm{pp} .+3 \mathrm{pl}$.

SANdground (J. H.), 1928. - Some new Cestodes and Nematodes parasites from Tanganyika territory. Proc. of the Boston Soc. of Nat. Hist., XXXIX, $131-150+$ pl. $16-21$.

SchNeider (A.), 1866. - Monographie der Nematoden, Berlin, $\mathrm{v}+357$ pp. $28 \mathrm{pl}$.

Schulz (R. Ed.), 1927. - Die Familie Physalopteride Leiper, 1908 (Nématod:s) und die Prinzipien ihrer Klassifikation. Samml. Helm. Arbeit. Prof. K. I. Skrjabin, Moscou, 287-312 + 1 pl. (En russe, résumé en allemand).

Seurat (L. G.), 1914. - Sur deux Physaloptères tétrahystériens des Reptiles. C.R. Soc. Biol., LXXVII, 433-436, fig. 1-5.

Seurat (L. G.), 1917. - Physaloptères des Reptiles du Nord-Africain. C.R. Soc. Biol., LXXX, 43-52, fig. 1-4.

Shiplex (A. E.), 1899. - A deseription of the Entozoa collected by Dr. Willey during his sejourn in the Western pacific. A. Willey's Zoological Results. Part. V (Cambridge University Press), 532-568, pl. LIV-LVI.

Skrjabin (K. I.), 1916. - Parasitic Trematodes and Nematodes collected by the expedition of Prof. V. Dogiel and 1. Sokolov in British East Africa. Expeditions of Prof. I. Dogiel and I. Sololov in Britisch East Africa. Zool. Results, Pétrograd, 157 pp. +10 pi. (en russe, résumé en anglais).

SPREHN (C. von), 1932. - Ueber einige von Dr. Eisentraut in Bolivien gesarnmelte Nematoden. Zool. Anzeig., Bd. 100, Heft 11/12, 273-284. 
Stiles (Ch. W.) et Hassall (A.), 1920. - Index-catalogue of medical and veterinary zoology. Subjects : Roundworms (Nematoda, Gordiacea, and Acanthocephala) and the Diseases they cause. Treasury department U.S. Pub. Health Ser., Bull. $\mathrm{n}^{\circ}$ 114, Washington, 886 pp.

Travassos (L.), 1920. - Contribuiçoes para o conhecimento da fauna helmintolojica brazileira. Mem. Inst. Osw. Cruz, XII, 66-77 + pl. 13-16.

WaLton (A. C.), 1927. - A revision of the Nematodes of the Leidy collections. Proc. Acad. Nat. Sc. Philadelphie, LXXIX, 49-163, fig. 1-79.

Watton (A. C.), 1932. - Physaloptera polydentata n. sp. J. Parasit., XVIII, 288-290, fig. $1 c$.

Institut de Parasitologie, Faculté de Médecine de Paris (Directeur : Professeur H. Gall.iard) 